\section{Desafios da Atenção Básica em Saúde: a experiência de Vila Mariana, São Paulo, Brasil}

\author{
Challenges in Primary Health Care: the experience \\ in Vila Mariana District, São Paulo, Brazil
}

\author{
1 Departamento de Medicina \\ Preventiva, Universidade \\ Federal de São Paulo, São \\ Paulo, Brasil.

\section{Correspondência} \\ W. V. Villela \\ Departamento de Medicina \\ Preventiva, Universidade \\ Federal de São Paulo. \\ Rua Borges Lagoa 1341, \\ São Paulo, SP \\ 04038-904, Brasil. \\ wilsa.vieira@terra.com.br
}

\begin{abstract}
This article presents the results of a case study aimed at identifying challenges for the delivery of primary health care in a health district in the city of São Paulo, Brazil, considering comprehensiveness as a central device for organizing work at this level of care. Five units in the district's primary care network were studied. We collected the data by observing work routines and flows, conducting interviews with managers and health professionals from different backgrounds, and through focus groups with family health teams. The analysis shows a mismatch between users' characteristics and their presumed needs and the heterogeneity of the health professionals' perceptions concerning their work. Lack of staff and time and difficulties with referrals were the main problems identified by interviewees.
\end{abstract}

Primary Health Care; Single Health System; Health Services

\author{
Wilza Vieira Villela 1 \\ Eliane Cardoso de Araújo ${ }^{1}$ \\ Sandra Aparecida Ribeiro 1 \\ Aloísio Punhagui Cuginotti 1 \\ Eliana Tiemi Hayana 1 \\ Francisco Carlos de Brito 1 \\ Luiz Roberto Ramos 1
}

\section{Introdução}

Desde 1978, com a realização da Conferência de Alma-Ata, a Atenção Básica à Saúde tem sido considerada um dos pilares da organização de qualquer sistema de saúde. Sendo o primeiro contato do usuário com o sistema de saúde, o nível básico de atenção à saúde tem um grande potencial de resolver parte significativa das queixas / demandas apresentadas 1 . Considere-se que, neste nível de atenção, a idéia de resolver não se restringe à elaboração de um diagnóstico de doença ou disfunção no corpo e oferta dos tratamentos correspondentes, incorporando, além do manejo específico dos agravos, ações de prevenção e promoção de saúde, identificação de necessidades que devem ser respondidas por outros serviços que não os da rede de saúde, e a referência do usuário a níveis mais complexos do sistema ${ }^{2}$. Assim, quase sempre vezes a atenção primária é capaz de solucionar a maioria dos problemas de saúde de grande da população ${ }^{3}$, a partir do atendimento das necessidades de saúde, o seguimento dos usuários, a oferta de atenção integral aos problemas mais freqüentes e a organização dos encaminhamentos necessários.

Para que o nível básico de atenção à saúde seja capaz de oferecer uma atenção integral é fundamental a interação ativa entre provedor e usuário ${ }^{4}$, sob forma de acolhimento - atitude do profissional e da equipe de receber, escutar e tratar de forma humanizada os usuários e suas 
demandas -, e cuidado - preocupação e responsabilidade pelo outro, o usuário 5 .

Esta particularidade da atenção básica à saúde - abarcar e buscar responder um grande conjunto de necessidades, por meio do acolhimento e do cuidado - tem sido incorporada à idéia de integralidade, na medida em que aponta para o entendimento de que necessidades de saúde não se remetem a necessidades de cuidados médicos. Necessidades de saúde devem ser compreendidas como os resultados de articulações singulares entre condições biológicas, sociais e psíquicas de um sujeito em um dado momento da vida. De acordo com Cecílio ${ }^{6}$, as necessidades de saúde podem ser organizadas em torno de quatro eixos: boas condições de vida, acesso às tecnologias capazes de melhorar e prolongar a vida, vínculos com a equipe e com os serviços de saúde e autonomia quanto ao modo de levar a vida. Esta compreensão ampla das necessidades de saúde deve orientar a construção do sentido de integralidade da atenção, fazendo corresponder a oferta de uma atenção integral à busca das respostas possíveis ao conjunto das necessidades de saúde percebidas pelo profissional em cada indivíduo à sua frente. Assim, a concretização da idéia de integralidade exige o reconhecimento de que o usuário que busca o serviço de saúde é um sujeito inserido numa complexa trama psicossocial, na qual sua queixa se inscreve e adquire um sentido particular e único.

A implementação de um modelo de atenção básica em saúde voltado para responder as necessidades de saúde dos diferentes sujeitos que constituem uma população, no Sistema Único de Saúde brasileiro, o SUS, não é simples. Exige uma organização ágil e eficiente de diferentes elementos, incluindo serviços de saúde e profissionais, que devem compor uma rede articulada de cuidados de modo a dar conta de um conjunto extenso e variado de sujeitos e demandas. Para responder a este desafio, o SUS define que os municípios devem organizar os serviços e atividades oferecidos à sua população de acordo com as necessidades identificadas e prioridades definidas localmente.

O funcionamento de uma rede de saúde com serviços para os diferentes níveis de atenção, a identificação de necessidades e definição de prioridades é particularmente complexo nas grandes metrópoles, onde fluxos migratórios trazem pessoas de origens sócio-culturais distintas, as diversas ocupações impõem horários e ritmos de trabalho variados e as pressões do cotidiano acarretam riscos específicos 7. Na cidade de São Paulo, por exemplo, cerca de 11 milhões de habitantes convivem com contrastes sociais e econômicos, diferentes formas de violência e uma crônica insuficiência da malha viária. Neste contexto, as desigualdades sociais e suas repercussões na saúde se fazem mais visíveis, ao mesmo tempo em que existe uma contínua produção de tecnologias biomédicas que pressionam profissionais e usuários em direção ao consumo crescente deste tipo de alternativas para a saúde 8 .

Há que se considerar que as idéias sobre a produção da saúde e da doença são construídas a partir de uma infinidade de referências e informações dispersas no espaço social. Situações percebidas pelos usuários como problemas de saúde, que produzem demandas aos serviços, e situações identificadas pelos profissionais como tal nem sempre coincidem. Isto implica a necessidade do diálogo ativo - acolhimento - entre provedor e usuário, e o reconhecimento, por parte de ambos, das possibilidades de resposta do serviço - o cuidado.

Conhecer o que os profissionais percebem como a vocação de um serviço de atenção básica à saúde permite aprimorar propostas voltadas para identificar necessidades de saúde expressas como demandas aos serviços, de modo a assegurar a integralidade da atenção.

Neste sentido foi realizado um estudo de caso incluindo as cinco unidades que compõem a rede de atenção básica do distrito de saúde da Vila Mariana, na cidade de São Paulo, visando mapear as concepções dos profissionais a respeito do seu trabalho neste nível de atenção à saúde.

\section{Abordagem metodológica}

O estudo tomou como referência a idéia de integralidade, princípio do SUS incorporado ao vocabulário da saúde pública brasileira como um conjunto de valores e características desejáveis em um sistema de saúde 9 .

Outrossim, considerou-se que as ações de saúde oferecidas para uma dada população devem levar em conta suas características próprias, na medida em que estas configuram um perfil de demanda e apontam estratégias que podem facilitar uma maior ou menor adesão às propostas dos serviços.

Para a coleta de dados foram desenvolvidos quatro instrumentos: um roteiro de entrevista para os gerentes, contemplando a descrição dos fluxos e rotinas de atendimento, as percepções das necessidades e demandas da população atendida e as concepções sobre qualidade e integralidade na atenção; um roteiro de entrevista para profissionais e um para grupos focais com as equipes de saúde da família abordando os mesmos temas sob o recorte de ações programáticas desenvolvidas nas unidades e as respecti- 
vas populações prioritárias - mulheres, idosos, e portadores de diabetes e hipertensão. Um último roteiro orientou a observação das atividades.

No período de dezembro de 2006 a junho de 2007 , foram realizadas 33 entrevistas com profissionais que atuam no atendimento direto à população, em número variável por unidade em função das disponibilidades, sendo assegurada em cada uma ao menos a participação de dois médicos, um enfermeiro, um auxiliar de enfermagem e um auxiliar técnico administrativo. No total foram entrevistados 11 médicos, incluindo clínicos, gineco-obstetras, homeopata, acupunturistas e pediatras; cinco enfermeiros; dois assistentes sociais, duas psicólogas, uma educadora, sete auxiliares de enfermagem e cinco auxiliares técnico-administrativos. Os cinco gerentes das unidades também foram entrevistados, e foram realizados três grupos focais com equipes de saúde da família. A observação dos serviços foi feita mediante sete visitas, ou seja, duas unidades receberam mais de uma visita. As entrevistas foram pré-agendadas e realizadas durante o período de trabalho do profissional, no dia e hora por ele definido, após apresentação do projeto e assinatura de termo de consentimento livre e esclarecido.

As entrevistas foram gravadas e posteriormente transcritas e as observações anotadas sistematicamente em diário de campo. A análise das entrevistas foi feita buscando-se identificar convergências e divergências nos discursos dos profissionais em relação a um dado tema. As anotações do diário de campo possibilitaram aprofundar o entendimento dos discursos dos entrevistados em cada uma das unidades, relacionando-os com a situação observada.

Embora as cinco unidades tivessem características distintas, a comparação entre as unidades não foi objeto deste trabalho, sendo, no entanto, levado em conta no momento da análise.

A realização do projeto contou com apoio do Conselho Nacional de Desenvolvimento Científico e Tecnológico (CNPq). O projeto foi aprovado pelos comitês de ética em pesquisa da Universidade Federal de São Paulo (UNIFESP) e da Secretaria de Higiene e Saúde do Município de São Paulo.

\section{Resultados}

\section{O território e os serviços estudados}

O subdistrito de saúde da Vila Mariana está localizado na região sudeste do Município de São Paulo. Conta com 306.437 habitantes, predominantemente de classe média ou média-alta e dis- põe de boa cobertura no suprimento de água, saneamento e coleta de lixo.

A população residente concentra-se na faixa etária de 20 a 49 anos. Em relação a outros bairros da cidade, a proporção de menores de 14 anos é menor, e maior a de pessoas acima de 50 anos. A partir dessa faixa etária o número de mulheres é significativamente mais alto que o dos homens. Em relação aos demais bairros da cidade o distrito da Vila Mariana exibe taxas menores de violência e desigualdade social. Existe uma concentração de serviços de saúde públicos, privados e universitários e o perfil de morbimortalidade caracteriza-se pelo predomínio de doenças ligadas ao processo de envelhecimento, sendo baixas as taxas de óbitos por causas evitáveis 10 .

Dentre as cinco unidades estudadas, uma é a unidade de saúde da família do distrito, e conta com quatro equipes. Duas das outras oferecem atendimento em acupuntura e uma destas também em homeopatia; outra conta com atendimento dermatológico. À exceção de uma das unidades que atua como referência em homeopatia e acupuntura e não faz atendimento a crianças, as demais realizam atendimento em clínica geral, gineco-obstetrícia, pediatria e procedimentos básicos de enfermagem. As quatro unidades básicas contam com atendimento em saúde mental realizado por psicóloga.

Todas desenvolvem ações de atenção à saúde reprodutiva, com acompanhamento pré- e pós-natal e referência para parto, orientação contraceptiva e grupo de planejamento familiar, e têm implantado o Programa Remédio em Casa. Algumas unidades realizam atividades físicas com idosos e portadores de hipertensão e diabetes, como grupos de caminhada e ginástica. À exceção da unidade de saúde da família, todas referem dificuldade em desenvolver atividades regulares para jovens, pela baixa freqüência deste grupo nas unidades e pouca adesão das escolas próximas para iniciativas conjuntas. Apenas a unidade de saúde da família realiza reuniões de equipe regularmente. As demais alegam que a necessidade de atender prontamente a demanda impede o cancelamento das agendas para reuniões. Esta falta de reuniões de equipe pode ser um obstáculo importante para a construção da integralidade, na medida em que esta exige a interação dos saberes e das práticas dos membros da equipe de trabalho.

Para matricular-se o usuário deve comprovar que reside ou trabalha na área de abrangência da unidade. O tempo de espera para obter a consulta é variável, em função da unidade e do tipo de atendimento. Três das unidades dispõem de um profissional da enfermagem ou do serviço social para fazer o acolhimento 
dos pacientes que chegam sem agendamento, mesmo os não matriculados. Nas outras duas o acolhimento é feito pela pessoa que atende na recepção. O encaminhamento para unidades mais complexas ou para realização de exames é feito por uma central de regulação, conectada às unidades por computador. Ressalte-se que, na perspectiva da integralidade, o acolhimento é uma tecnologia específica, que não se reduz à recepção e encaminhamento dos usuários 5 . A superposição entre acolhimento e a recepção pode assim dificultar que essa tecnologia atenda as suas finalidades mais amplas.

A população atendida, exceto na unidade de saúde da família, compõe-se primordialmente de pessoas que trabalham na região, em especial mulheres empregadas domésticas e homens empregados na construção civil, com idade acima de quarenta anos. Este perfil de usuários - que não residem em torno das unidades - contrasta com um dos sentidos atribuídos à idéia de integralidade, que fala de adscrição da clientela, vínculo e seguimento dos usuários. Este é um desafio colocado hoje em várias metrópoles, onde as pessoas se deslocam por longas distâncias em função do trabalho e não acessam os recursos próximos da sua residência, requerendo novos arranjos organizacionais para promover a saúde e organizar serviços 11.

Existe um grupo menos numeroso, composto por pessoas idosas ou aposentadas, que moram próximas às unidades e as utilizam para fazer controles de saúde, tomar vacinas ou obter medicação, embora tenham consultas pelos planos de saúde, numa utilização combinada de serviços de saúde públicos e privados já apontada por outros autores 12 .

\section{A percepção dos profissionais sobre o seu trabalho}

Um primeiro tópico investigado com gerentes e profissionais foi a sua percepção quanto à organização do cuidado - modo como os agentes do trabalho percebem a sua atividade e se organizam para atender às demandas dos usuários. Foi possível apreender que, para os gerentes, a idéia da integralidade se confunde com a idéia mesma de cuidar, entendida como o conhecimento das dimensões do cotidiano do usuário e a elaboração de respostas adequadas: "é reconhecer, acolher, compreender as necessidades mesmo que não possa resolver". No entanto, os gerentes são unânimes em afirmar o cuidado, assim definido, é de difícil concretização, dadas a falta de funcionários e de tempo para reuniões e outras atividades necessárias à construção de uma abordagem interdisciplinar. Como refere uma entrevistada,
“Existe grupo e não equipe. O medico faz tratamento, prioriza a consulta, a enfermeira faz prevenção, monitora os grupos, o agente comunitário faz educação em saúde". A falta de integração e as dificuldades em trabalhar em equipe limitam a possibilidade de "articular os diferentes olhares sobre o mesmo problema”, pressuposto da proposta de integralidade em saúde.

Entre os profissionais as idéias referentes à organização do cuidado já não encontram tanta unanimidade. Alguns médicos, por exemplo, percebem a sua prática como essencialmente clínica, e não valorizam a resolutividade que podem ter no nível básico de atenção: “a gente como Clínico tem que dar uma de especialista, é difícil você fazer encaminhamento, é pelo menos um ano para ele ser avaliado pelo colega". Para estes profissionais, problemas relacionados à estrutura e organização do sistema, como a cobrança de produtividade e a falta de vagas para a realização de exames e consultas especializadas são os principais obstáculos para o cuidado integral, restringindo o papel resolutivo da atenção básica e suas possibilidades de atender as demandas que chegam até ela.

Na perspectiva dos médicos, a unidade básica de saúde atua como uma "ponte amenizando os bloqueio e entraves, facilitando e encurtando o caminho do paciente pra ser ouvido e atendido".

A idéia de cuidado também é associada por alguns profissionais, médicos e não médicos, como uma dimensão educativa das praticas de saúde, o que tem sido defendido por alguns estudiosos do tema ${ }^{13}$. Entretanto, as ações de promoção e prevenção, tão importantes na construção da idéia do cuidado e de integralidade, não são percebidas como tendo a mesma importância que a prescrição de medicamentos, e sim como uma alternativa de menor valor, na falta do remédio, considerado ideal, como mostra a seguinte afirmação, sobre o atendimento de mulheres no climatério: "Em termos de SUS, se a mulher não puder comprar, ela tem como alternativa a mudança do estilo de vida". Esta ambigüidade em relação ao próprio trabalho e às diretrizes do SUS voltadas para a produção da saúde, e não apenas para o cuidado com os doentes, aponta a dificuldade de alguns profissionais perceberem a potencialidade do nível básico de atenção e das ações que manejam tecnologias leves na resolução dos problemas de saúde mais prevalentes, em que pese a literatura em contrário 14.

Os profissionais das equipes de saúde da família afirmam que a maior proximidade com o usuário possibilita aprofundar o entendimento da relação entre a queixa e o seu cotidiano e, a partir disto, desenvolver ações de prevenção e promoção da saúde. Contudo existe uma ên- 
fase na consulta médica posta pelo usuário e reforçada na exigência de produtividade nesta modalidade de atenção que não ocorre em relação às práticas educativas, fazendo com que os profissionais não médicos sintam-se menos reconhecidos.

A centralidade do atendimento médico gera uma grande demanda por consultas, o que para os gerentes e demais profissionais prejudica a qualidade do trabalho: "O tempo limitado da consulta médica compromete o vinculo que se busca construir em outros espaços. Dificulta também a comunicação entre o paciente e o medico". Segundo estes "o que o paciente mais valoriza em uma atenção integral é o vinculo", "é se colocar no lugar do paciente vivenciar o lado dele”.

Para os assistentes sociais, psicólogos e educadores, a sua prática se situa num ponto intermediário entre as perspectivas sobre cuidado expressas pelos gerentes, mais genéricas e abstratas, e o olhar essencialmente clínico dos médicos. Suas falas apontam o seu trabalho como uma mediação entre as necessidades de saúde percebidas e a demanda por consulta médica: "a pediatra foca mais o ponto de vista objetivo do aleitamento e eu fico tentando criar um vínculo com ela e com as gestantes...", revelando a potencialidade das equipes multiprofissionais no âmbito da atenção básica.

Na unidade de saúde da família são ainda referidos, como desafios, o número excessivo de famílias por equipe, a carência de uma rede de suporte para os usuários e de apoio psicológico para os profissionais, a necessidade de atividades regulares de capacitação e a insuficiência da formação dos profissionais para a promoção de saúde. Os agentes comunitários apontam ainda dificuldades na identificação das necessidades de saúde dos usuários, no encaminhamento de soluções possíveis. Distorções na compreensão das atribuições dos agentes pelos usuários foram também comentadas como um entrave à efetividade do seu trabalho.

\section{As atividades oferecidas e as necessidades de saúde percebidas}

Buscou-se compreender a oferta da atenção integral tendo como referência as características da clientela, suas demandas e necessidades percebidas.

Em relação ao atendimento de mulheres, maior grupo populacional nas unidades estudadas, considerou-se a Política Nacional de Atenção à Saúde da Mulher (PNAISM), que aponta para outras ações além da atenção à saúde reprodutiva, como a busca ativa das vivências de violência e a abordagem da sexualidade 15 .
As usuárias das unidades estudadas tem, em média, idade igual ou maior que quarenta anos, e segundo as entrevistas, apresentam como queixas “a hipertensão (...) e os problemas de alteração psicológica, estresse, cansaço, porque elas trabalham com muita pressão das patroas, vêm aqui porque ficaram nervosas...”. Paradoxalmente, as ofertas para esta população estão focadas na assistência pré-natal e no atendimento ginecológico. Quando, de modo espontâneo, surgem queixas relacionadas à sexualidade, à saúde mental ou à violência doméstica, na falta de fluxos ou protocolos estruturados, e de espaços com maior privacidade para sua abordagem, as respostas são dadas de acordo com o profissional que atende. Para situações de violência doméstica, por exemplo, "eu vou discutir o caso com a Psicologia, ou eu passo para a gente atender juntas, ou ela já atendeu e resolveu, dependendo do caso ela já faz ela mesma". A violência contra mulheres não é reconhecida como objeto de trabalho ${ }^{16}$, sendo considerado que algumas mulheres tem a violência incorporada no seu cotidiano: "ela chega em casa o marido dá um tapa, chega no trabalho o patrão dá na cabeça dela, ela pensa aquilo é normal, ela pensa, dá para agüentar, eu preciso do dinheiro".

A estrutura física das unidades e o fluxo do atendimento oferecem poucas condições para a abordagem de questões sensíveis, como sexualidade ou violência. Como nem sempre a usuária se sente à vontade para apresentar este tipo de demanda na sala de recepção, aberta ao público, quem vive estes problemas deve marcar consulta com o médico ou a psicóloga, que nem sempre podem atender de imediato. Isto mantém a violência invisível nos serviços, alimentando o círculo vicioso onde nem o serviço nem as usuárias tomam a violência doméstica como uma questão de saúde a ser enfrentada: Como refere uma entrevistada: "elas não falam, não chegam e falam apanhei, elas falam entre elas, mas não expõem o problema para nós resolvermos. Quando eu vou perguntar para uma delas, fica com vergonha, fala só o necessário...”.

Em contrapartida, as agentes comunitárias de saúde, que com freqüência se defrontam com situações de violência em suas visitas domiciliares, sentem-se despreparadas para qualquer abordagem, tendendo a não intervir.

O cuidado de mulheres portadoras de hipertensão e diabetes, que também constituem a maioria neste tipo de atendimento não é parte da atenção à saúde integral das mulheres. Ao contrário, estas usuárias são atendidas a partir de protocolos que não consideram que viver com um agravo crônico à saúde pode ser distinto para homens e para mulheres 17 , fragmentação que 
corresponde ao modo como estão organizadas as políticas no âmbito federal. A reprodução, em nível local, de propostas nacionais contraditórias aponta a dificuldade de adequação de diretrizes gerais às características concretamente exibidas pela população, o que pode se constituir em um obstáculo para a oferta de uma atenção integral voltada para dar respostas mais amplas às necessidades de saúde percebidas e identificadas.

No atendimento dos portadores de doenças crônicas, a dificuldade referida são os encaminhamentos para níveis mais complexos de atenção, além da pequena adesão ao programa do governo de entrega de medicamentos em casa. Aparentemente os usuários sentem-se mais seguros retirando o remédio na unidade de saúde, e assim o programa não atinge a cobertura estabelecida pelo gestor, gerando cobranças à equipe. A falta de funcionários para realizar as atividades previstas pelo programa de atenção aos idosos também é citada, bem como de um melhor planejamento das ações voltadas aos portadores de hipertensão e diabetes. Segundo uma entrevistada, o esforço em planejar em equipe as atividades para este grupo poderia ser oportuno: “deveria fazer uma reunião pelo menos uma vez por mês. Se a gente atender bem os diabéticos, os hipertensos, outras coisas também melhoram, muito, muito, muito, pois uma coisa leva a outra".

As descontinuidades no seguimento dos portadores de doenças crônicas, que trabalham na região e residem em bairros afastados, sua pouca vinculação ao serviço e baixa adesão ao tratamento e às ações de promoção de saúde propostas é motivo de frustração para alguns profissionais das unidades básicas. Em contrapartida, na unidade de saúde da família o que aparece, mais uma vez, é a sensação de que o usuário não reconhece a importância dessas atividades, realizadas pelo agente. Ou seja, tanto para quem trabalha dentro da unidade como para quem vai até o usuário, existe a percepção de que o usuário valoriza mais o trabalho do médico e prescrição de medicamentos, embora haja por parte dos agentes comunitários ou auxiliares de enfermagem, que a necessidade de capacitação específica para ações educativas e para a abordagem do processo de envelhecimento pode contribuir para esta desvalorização 18 .

As dificuldades para o atendimento aos idosos recaem sobre questões já apontadas: número excessivo de atendimentos, treinamento insuficiente das equipes, falta de integração com centros de especialidades para referência, falta de protocolos específicos. Assim, o atendimento das necessidades de saúde deste segmento etário mais uma vez depende do profissional, como referem as entrevistadas: "quando chegam velhi- nhos aqui, eu não marco consulta, eu já pego e na hora já faço o cartão. A pessoa viveu 70 e tarará 80 anos e esperar mais para que?". "A gente dá prioridade para aquele idoso que está com alguma deficiência que a gente visualiza, que está com uma muleta ou na cadeira de rodas". Vale lembrar que já existem modelos exitosos de capacitação de equipes em Saúde Integral do Idoso, que, no entanto, precisam ser expandidos de modo a atender à crescente demanda de profissionais buscando capacitação para lidar com o envelhecimento 19 .

\section{Discussão}

A estratégia de obter informações a partir de atores que desempenham funções distintas no processo de trabalho, e de complementá-las com observações in loco mostrou-se adequada aos propósitos desta investigação.

Embora não fosse buscada a comparação entre a unidade de saúde da família e as demais unidades, chamou atenção a semelhança dos problemas identificados pelos profissionais das unidades básicas de saúde e da unidade de Saúde da Família, sugerindo que, em alguns cenários, a menor ou maior efetividade de uma proposta de atenção básica em saúde está relacionada a outras circunstâncias que não os modelos de atenção adotados.

A adequação das ofertas à população a quem se destina é um desafio, dado que as políticas de saúde se dirigem prioritariamente às populações mais vulneráveis. Em situações como a estudada, a maioria dos usuários trabalhada na região, mas vive distante e muitas vezes são oriundos de contextos sócio-culturais distintos daqueles onde habitam ou trabalham. Assim, nem sempre conseguem auferir os benefícios de modelos de atenção calcados na idéia de vínculo, seguimento, agendamento e práticas voltadas para mudanças de estilos de vida. Seriam necessários modelos mais flexíveis de organização dos serviços que permitissem a vinculação ao serviço tendo em conta estas particularidades e os diferentes discursos e representações do provedor e do usuário sobre corpo, saúde e doença ${ }^{20}$, bem como sistemas de referência horizontais que garantissem a continuidade do atendimento em quaisquer regiões da cidade, para os casos de mudança de emprego ou moradia.

A compatibilização entre ofertas e características da clientela não se restringe aos fluxos e rotinas, devendo incluir as atividades programadas e protocolizadas, de modo a atender melhor as necessidades de saúde sentidas ou percebidas na população. Por exemplo, embora a 
atenção ao ciclo gravídico puerperal seja fundamental, em locais onde a clientela que se utiliza dos serviços está fora do período reprodutivo, outras ações se tornam igualmente prioritárias para as mulheres. O mesmo pode ser dito em relação às ofertas feitas para idosos, também mulheres em sua maioria, que enfrentam problemas biopsicossociais específicos relacionados ao envelhecimento, e que deveriam ser objeto de ações protocolizadas visando maior adesão e resolutividade. Ao mesmo tempo, embora havendo protocolos para os usuários com hipertensão e/ou diabetes mellitus, há alto percentual de faltas nas consultas e com freqüência ocorrem situações de descontrole pressórico ou glicêmico, sem que haja uma correspondente agilidade do sistema no encaminhamento para serviços de maior complexidade. A frágil articulação com os serviços que dariam continuidade ao atendimento de doentes crônicos, ao lado da reiterada queixa dos profissionais das dificuldades de exames e consultas com especialistas, ratifica o pequeno reconhecimento e valorização do potencial resolutivo do nível básico de atenção à saúde, gerando demandas dos profissionais e dos usuários de consumo de tecnologias mais duras 21 .

Os discursos dos profissionais a respeito de cuidado, acolhimento, e integralidade, não conseguem se concretizar nas suas práticas. A razão deste descompasso, segundo eles, é a organização do serviço e a diretriz para que as portas dos serviços estejam sempre abertas, o que gera uma grande demanda, e o modelo de assistência, que não incorpora tecnologias mais sofisticadas neste nível de atenção, produzindo insatisfação nos profissionais e usuários, e o uso repetido da unidade. Esta é uma situação delicada, pois não se podem recusar à clientela os benéficos dos exames e consultas especializadas, do mesmo modo como não se pode negar a efetividade de um atendimento cuidadoso, realizado sem pressa, a partir do diálogo com o profissional, com garantia de seguimento e de atendimento não agendado em caso de intercorrência 22. Assim, trata-se de encontrar o difícil equilíbrio entre a boa prática clínica e a oferta dos recursos diagnósticos e terapêuticos disponíveis no contexto de uma demanda ainda pouco familiarizada à oferta de serviços com base na integralidade e na perspectiva das necessidades de saúde 23 .

A universalização da atenção à saúde que orienta o SUS deve enfrentar o desafio de ofertar ações de saúde a uma população heterogênea e marcada pela desigualdade social, buscando, ademais, contribuir para que os sujeitos se sintam mais potentes para gerir suas vidas. Deve também assimilar as inovações tecnológicas continuamente postas à disposição dos profissionais e usuários do sistema. Isto exige um processo constante de análise crítica por parte dos gerentes e gestores, criação de protocolos específicos, capacitação e supervisão dos recursos humanos 24 .

No distrito de saúde estudado os recursos humanos são insuficientes em número e os profissionais têm poucas oportunidades para construir um trabalho em equipe. Suas práticas não se dirigem às necessidades de saúde do usuário, comprometendo a integralidade na atenção. Isto sugere a importância do desenvolvimento de habilidades dos profissionais do SUS para a prática da atenção integral durante a sua formação e ao longo do exercício profissional, a partir de modelos de organização de serviços mais dialógicos e participativos 25 .

A adscrição da clientela, que em geral facilita o vínculo do usuário com o serviço, talvez não seja a melhor alternativa de atenção à saúde onde a população que se utiliza dos serviços não reside na região. No entanto, a busca de modelos de organização de serviços compatíveis com as características dos seus usuários não é simples.

A necessidade de adequar ofertas inclui tanto as rotinas de trabalho quanto os conteúdos. De fato, a adoção de protocolos para o atendimento de gestantes e portadores de hipertensão de diabetes aumenta a efetividade do trabalho. $\mathrm{O}$ mesmo pode ser dito em relação à oferta de uma gama variada de atividades de promoção de saúde e prevenção de doenças junto aos idosos. No entanto, é necessário que estas ofertas não sejam consideradas "atendimento de segunda categoria” pelos usuários ou pelos provedores. Conforme enfatizam Motta \& Aguiar 26 (p. 366), "o conhecimento do processo de envelhecimento (...) reitera a importância do trabalho preventivo (...) sendo necessário incluir, na rotina dos serviços e na formação dos profissionais "conteúdos sobre envelhecimento, saúde do idoso, trabalho em equipe e noção de saúde ampliada em quase todas as disciplinas ao longo do curso".

Os problemas de saúde que emergem com o aumento do tempo de vida e as crescentes vicissitudes do viver em grandes cidades - estresse, isolamento, violência - que constituem agravos importantes à saúde, exigem um investimento em ações de promoção e prevenção, e na incorporação de novas possibilidades diagnósticas e terapêuticas úteis e custo-efetivas. Como os resultados deste trabalho mostram, esta é uma questão ainda não completamente equacionada nos serviços estudados. 


\section{Considerações finais}

Integralidade e atenção integral à saúde são idéias que têm inspirado propostas criativas de organização de sistemas e modelos de atenção nas quais o vínculo entre provedor e usuário é valorizado 27 , bem como a capacidade de resposta às suas necessidades de saúde. Os resultados apresentados mostram que as concepções dos profissionais nos serviços estudados sobre o que seria uma "boa prática" não são homogêneas, variando em ênfases sobre procedimentos clínicos, acolhimento da demanda, abordagens interdisciplinares e trabalho em equipe. O modo como os serviços e o sistema estão organizados é a principal razão apontada para que o ideal de boa prática, qualquer seja, não se concretize. É necessário mais diálogo com profissionais e população sobre as potencialidades e limites do trabalho em saúde neste nível de atenção, de modo a evitar que a atenção básica em saúde seja percebida como uma intermediação entre o sofrimento tangível do usuário e um suposto bem estar obtido por meio de tecnologias pouco acessíveis.

\section{Resumo}

Este artigo apresenta resultados de estudo de caso visando identificar desafios na oferta de atenção básica à saúde em um distrito de saúde da cidade de São Paulo, Brasil, considerando integralidade como dispositivo organizador do trabalho neste nível de atenção. Foram estudadas as cinco unidades que compõem a rede de atenção básica à saúde no distrito considerado. Os dados foram coletados mediante observação dos fluxos $e$ rotinas de atendimento, realização de entrevistas com gerentes e profissionais de diferentes formações e ainda realização de grupos focais com equipes de saúde da família. A análise mostra um descompasso entre as características da clientela e suas necessidades presumidas e as ofertas dos serviços, e heterogeneidade nas percepções dos profissionais sobre seu trabalho. Falta de profissionais, de tempo, e dificuldades para encaminhamentos foram os principais problemas apontados pelos entrevistados.

Atenção Primária à Saúde; Sistema Único de Saúde; Serviços de Saúde

\section{Colaboradores}

Todos os autores participaram igualmente de todas as fases de desenvolvimento do projeto e elaboração do manuscrito.

\section{Agradecimentos}

Os autores agradecem ao Conselho Nacional de Desenvolvimento Científico e Tecnológico (CNPq, processo 403258/2005/7) o apoio recebido para a realização do projeto. 


\section{Referências}

1. World Health Organization. Primary health care. http://www.who.int/topics/primary_health_care/ en/ (acessado em 12/Abr/2008).

2. Health Canada. About primary health care. http:// www.hc-sc.gc.ca/hcs-sss/prim/about-aproposeng.php\#4 (acessado em 12/Abr/2008).

3. Starfield B. The primary solution. Boston Review 2005; http://www.bostonreview.net/BR30.6/ starfield.html (acessado em 18/Set/2008).

4. Teixeira RR. Humanização e atenção primária em saúde. Ciênc Saúde Coletiva 2005; 10:585-97.

5. Camargo Jr. KR, Campos EMS, Bustamante-Teixeira MT, Mascarenhas MTM, Mauad NM, Franco TB, et al. Avaliação da atenção básica pela ótica político-institucional e da organização da atenção com ênfase na integralidade. Cad Saúde Pública 2008; 24 Suppl 1:S58-68.

6. Cecílio LCO. As necessidades de saúde como conceito estruturante na luta pela integralidade e equidade na atenção à saúde. In: Pinheiro $\mathrm{R}$, Mattos R, organizadores. Os sentidos da integralidade na atenção e no cuidado à saúde. Rio de Janeiro: Instituto de Medicina Social, Universidade do Estado do Rio de Janeiro/ABRASCO; 2001. p. 113-27.

7. Machado CV, Lima LD, Viana LS. Configuração da Atenção Básica e do Programa Saúde da Família em grandes municípios do Rio de Janeiro, Brasil. Cad Saúde Pública 2008; 24 Suppl 1:S42-57.

8. Baron RJ, Cassel CK. New physician roles need new payment models. JAMA 2008; 299:1595-7.

9. Mattos RA. Os sentidos da integralidade: algumas reflexões acerca de valores que merecem ser defendidos. In: Pinheiro R, Mattos R, organizadores. Os sentidos da integralidade na atenção e no cuidado à saúde. Rio de Janeiro: Instituto de Medicina Social, Universidade do Estado do Rio de Janeiro/ ABRASCO; 2001. p. 39-65.

10. Prefeitura Municipal de São Paulo. Município em mapas. http://www9.prefeitura.sp.gov.br/sempla/ $\mathrm{mm} /$ (acessado em 19/Set/2008).

11. Akerman M, Duhl L, Bógus CM. A questão urbana e a saúde: impactos e respostas necessárias. In: Castro A, Malo M, organizadores. SUS: ressignificando a promoção da saúde. São Paulo: Editora Hucitec/Organização Pan-Americana da Saúde; 2006. p. 119-32.

12. Pessoto UC, Heimann L, Boareto R, Castro IEN, Kayano J, Ibanhez L, et al. Desigualdades no acesso e utilização dos serviços de saúde na Região Metropolitana de São Paulo. Ciênc Saúde Coletiva 2007; 12:351-62.
13. Machado MFAF, Monteiro EMLM, Queiroz DT, Vieira NFC, Barroso MGT. Integralidade, formação de saúde, educação em saúde e as propostas do SUS - uma revisão conceitual. Ciênc Saúde Coletiva 2007 ; $12: 335-42$.

14. Souza ECF, Vilar RLA, Rocha NSPD, Uchoa AC, Rocha PM. Acesso e acolhimento na atenção básica: uma análise da percepção dos usuários e profissionais de saúde. Cad Saúde Pública 2008; 24 Suppl 1:S100-10.

15. Ministério da Saúde. Política de atenção à saúde integral da mulher. Brasília: Ministério da Saúde; 2004.

16. D'Oliveira AFPL, Kiss LB, Schraiber LB. Possibilidades de uma rede intersetorial de atendimento a mulheres em situação de violência. Interface Comun Saúde Educ 2007; 11:485-501.

17. Doyal L. Sex, gender and health: the need for a new approach. BMJ 2001; 323:1061-3.

18. Piccini RX, Facchini LA, Tomasi E, Thumé E, Silveira DSS, Siqueira VF, et al. Necessidades de saúde comuns aos idosos: efetividade na oferta e utilização em atenção básica à saúde. Ciênc Saúde Coletiva 2006; 11:657-67.

19. Motta LB, Caldas CP, Assis M. A formação de profissionais para atenção integral à saúde do idoso: a experiência interdisciplinar do NAI-UNATI/UERJ. Ciênc Saúde Coletiva 2008; 13:1143-51.

20. Pereira PPG. O terror e a dádiva. Goiânia: Editora Vieira; 2004.

21. Parker A. Health technology and primary health care. Soc Sci Med [Med Econ] 1978; 12:29-41.

22. Reuben BD. Better care for older people with chronic diseases: an emerging vision. JAMA 2007; 298:2673-4.

23. Campos CMS, Bataiero MO. Necessidades de saúde: uma análise da produção científica brasileira de 1990 a 2004. Interface Comum Saúde Educ 2007; 11:605-18.

24. Campos GWS. Reforma política e sanitária: a sustentabilidade do SUS em questão? Ciênc Saúde Coletiva 2007; 12:351-62.

25. Araújo D, Miranda MCG, Brasil S. Formação de profissionais de saúde na perspectiva da integralidade. Rev Baiana Saúde Pública 2007; 31 Suppl 1: 20-31.

26. Motta LB, Aguiar AC. Novas competências profissionais em saúde e o envelhecimento populacional brasileiro: integralidade, interdisciplinaridade e intersetorialidade. Ciênc Saúde Coletiva 2007; 12:363-72.

27. Mattos RA. A integralidade na prática (ou sobre a prática da integralidade). Cad Saúde Pública 2004; 20:1411-6.

Recebido em 15/Mai/2008

Versão final reapresentada em 16/Dez/2008

Aprovado em 21/Jan/2009 